\title{
Synthesis of Polyamides via in-situ Polycondensation
}

\author{
Naoya Ogata and Hozumi Tanaka \\ Department of Chemistry, Sophia University, 7 Kioi-cho, \\ Chiyoda-ku, Tokyo 102, Japan.
}

(Received September 7, 1976)

\begin{abstract}
KEY WORDS Polycondensation / Polyamide / Nylon Salt Aryl
Sulfite /
\end{abstract}

It is well known ${ }^{1}$ that polyamides can be obtained under mild conditions by using active diesters such as phenyl esters. In this case, the isolation of the active diesters is necessary because of the purification. On the other hand, ary sulfites are found ${ }^{2}$ to be effective for synthesizing the active diesters in a quantitative yield by reacting aryl sulfites with carboxylic acids in the presence of a base such as pyridine or imidazole. Therefore, it is expected that polyamides may form under mild conditions when nylon salts from dicarboxylic acids and diamines are subjected to the reaction in the presence of pyridine or imidazole together with a stiochiometric amount of aryl sulfites, since active diesters may form in-situ in the solution and the polycondensation of the active diesters may follow. This paper describes the synthesis of polyamides via in-situ polycondensation by using aryl sulfites.

$$
\begin{gathered}
\mathrm{HOOC}-\mathrm{R}-\mathrm{COOH}+\mathrm{NH}_{2}-\mathrm{R}^{\prime}-\mathrm{NH}_{2} \frac{\stackrel{\mathrm{PhO}-\mathrm{I}}{\mathrm{S}}-\mathrm{OPh}}{\begin{array}{c}
\text { Pyiridine or } \\
\text { Imidazole }
\end{array}}\left[\mathrm{PhOOC}-\mathrm{R}-\mathrm{COOPh}+\mathrm{NH}_{2}-\mathrm{R}^{\prime}-\mathrm{NH}_{2}\right] \\
\longrightarrow+\mathrm{CO}-\mathrm{R}-\mathrm{CONH}-\mathrm{R}^{\prime}-\mathrm{NH}++2 \mathrm{PhOH}+\mathrm{H}_{2} \mathrm{O}+\mathrm{SO}_{2}
\end{gathered}
$$

\section{EXPERIMENTAL}

Diphenyl, di-( $p$-nitrophenyl) or cathecol sulfites were prepared by reacting thionyl chloride with corresponding phenols in refluxing monochlorobenzene..$^{3,4}$ Nylon salts from dicarboxylic acids and diamines were prepared by mixing methanol solutions of dicarboxylic acids and diamines, followed by filtration and recrystallization in aqueous methanol solution. Polycondensation reaction was carried out as follows: nylon salts or amino acids were suspended in dimethylformamide (DMF) in a desired concentration and a given amount of diphenyl sulfite and imidazole was added to the solution. When pyridine was used as a solvent, imidazole was not added. The whole solution was kept at room temperature or $60^{\circ} \mathrm{C}$ with a vigorous stirring in a given period. Then, the solution was poured into excess acetone and precipitated polymers were collected by filtration, washed with water and acetone and dried.

The polymers obtained were idintified as corresponding polyamides by infrared and elemental analyses. Solution viscosities of the polyamides were measured in the concentration of $0.5 \mathrm{~g} /$ $100 \mathrm{ml}$ of $96 \%$ sulfuric acid at $30^{\circ} \mathrm{C}$.

\section{RESULTS AND DISCUSSION}

Table I summarizes results of the polycondensation of various nylon salts or amino acids in the presence of diphenyl sulfite in dimethylformamide (DMF) or pyridine (Py). It is seen in Table I that imidazole was superior to pyridine as a base since an insoluble polymer was 
Table I. Polycondensation of nylon salts or amino acids in the presence of phenyl sulfite ${ }^{a}$

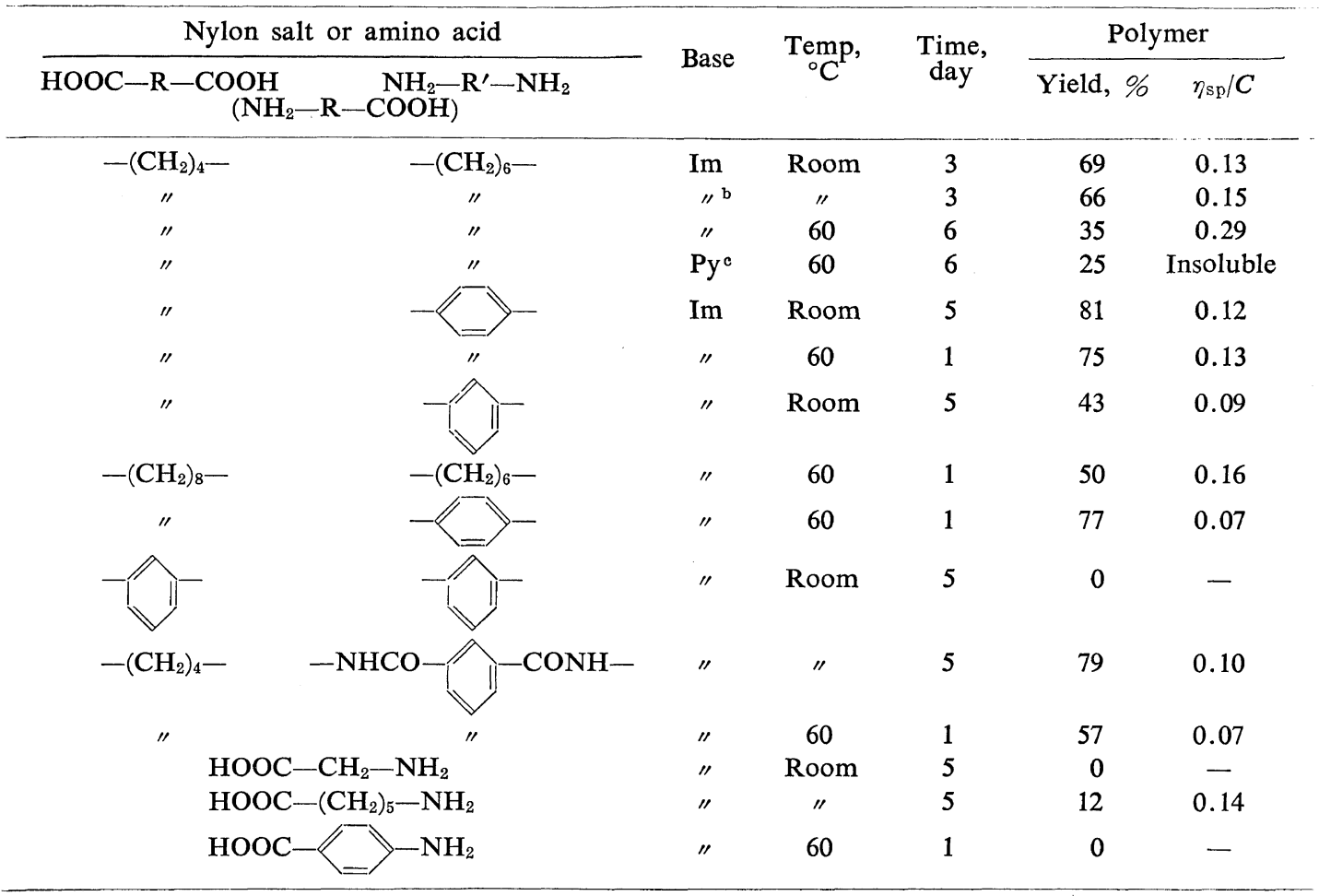

a Polycondensation was carried out by using $0.0025 \mathrm{~mol}$ of nylon salts or amino acids in the presence of $0.005 \mathrm{~mol}$ of phenyl sulfite and imidazol in $10 \mathrm{ml}$ of DMF.

b The amount of imidazole was reduced to $0.0025 \mathrm{~mol}$.

c $10 \mathrm{ml}$ of pyridine was used as a solvent.

formed in pyridine. Higher temperatures rather reduced polymer yields and thus room temperature was preferable. The combination of adipic acid and hexamethylenediamine produced a corresponding polyamide in about $70-\%$ yield and the solution viscosity of the polyamide was not satisfactory. This might be ascribed to an insufficient conversion of carboxylic acid to a corresponding active ester. Aromatic diamines such as $p$ - or $m$-phenylenediamines also produced the corresponding polyamides in a good yield in comparison with hexamethylenediamine, while aromatic dicarboxylic acids such as isophthalic acid did not yield any polyamides at all. Isophthalic acid dihydrazide could also produce a corresponding polyhydrazide from adipic acid by means of the same method. On the other hand, amino acids did not produce polyamides in the presence of phenyl sulfite and imidazole.
Therefore, the acidity or reactivity of carboxylic acid may be related to the formation of the active diesters by phenyl sulfite.

It is well known that $p$-nitrophenyl ester is easily subjected to a nucleophilic replacement by amine, in comparison with phenyl ester. Therefore, various sulfites including di-( $p$-nitrophenyl) sulfite, cathecol sulfite, or benzenesulfonyl chloride were investigated in terms of the catalytic activity for the polycondensation of nylon salts or amino acids. Table II summarizes the results of the polycondensation by these sulfites. As was expected, di-( $p$-nitrophenyl $)$ sulfite initiated the polycondensation of nylon 66 salt (adipic acid/hexamethylenediamine) to form polyamide in higher yield than diphenyl sulfite. However, fully aromatic polyamides were not obtained even by di-( $p$-nitrophenyl $)$ sulfite. Cathecol sulfite did not initiated the 
Table II. Polycondensation of nylon salts or amino acids by various sulfites

\begin{tabular}{|c|c|c|c|c|c|c|c|c|}
\hline \multirow{2}{*}{ Sulfite } & \multicolumn{2}{|c|}{ Monomer } & \multirow{2}{*}{ Base } & \multirow{2}{*}{ Solvent } & \multirow{2}{*}{$\underset{{ }^{\circ} \mathrm{C}}{\mathrm{Tem}}$} & \multirow{2}{*}{ Time, } & \multicolumn{2}{|c|}{ Polymer } \\
\hline & \multicolumn{2}{|c|}{$\left(\mathrm{NH}_{2}-\mathrm{R}-\mathrm{COOH}\right)$} & & & & & Yield, $\%$ & $\eta_{\mathrm{sp}} / C$ \\
\hline$=>-\mathrm{O}-)_{2} \mathrm{SO}$ & $-\left(\mathrm{CH}_{2}\right)_{4}-$ & $-\left(\mathrm{CH}_{2}\right)_{6}-$ & $\operatorname{Im}$ & DMF & Room & 20 & 87 & 0.10 \\
\hline " & $"$ & " & " & " & 60 & 24 & 50 & 0.07 \\
\hline " & " & $\mathrm{CO}-\langle=\mathrm{CONH}-$ & " & " & 60 & 24 & 60 & 0.04 \\
\hline " & \multicolumn{2}{|c|}{$\mathrm{NH}_{2}-\langle=\mathrm{COOH}$} & " & " & 60 & 24 & 0 & - \\
\hline$-\mathrm{O}>\mathrm{SO}$ & $-\left(\mathrm{CH}_{2}\right)_{4}-$ & $-\left(\mathrm{CH}_{2}\right)_{6}-$ & " & " & 60 & 24 & 0 & - \\
\hline$"$ & " & " & " & " & 60 & 24 & 0 & - \\
\hline$-S$ & " & " & Py & Py & Room & 41 & 77 & 0.22 \\
\hline " & " & " & $\operatorname{Im}$ & $\mathrm{THF}^{\mathrm{b}}$ & Room & 40 & 0 & - \\
\hline$"$ & $-\left(\mathrm{CH}_{2}\right)_{8}-$ & " & $\mathrm{H}_{3} \mathrm{PO}_{4}$ & Py & Room & 24 & 90 & 0.06 \\
\hline " & \multirow{2}{*}{\multicolumn{2}{|c|}{$\begin{array}{c}\mathrm{NH}_{2}-\mathrm{CH}_{2}-\mathrm{COOH} \\
\text { " }\end{array}$}} & Py & Py & Room & 14 & 16 & - \\
\hline " & & & Py & Py & Room & 41 & 75 & 0.08 \\
\hline
\end{tabular}

a Polycondensation was carried out by using $0.0025 \mathrm{~mol}$ of nylon salts in the presence of $0.005 \mathrm{~mol}$ of sulfites and base in $10 \mathrm{~m} l$ of solvent.

b Tetrahydrofuran.

- $20 \mathrm{~m} l$ of pyridine was used. 
polycondensation at all, since cathecol sulfite is stable enough to react with carboxylic acid, while benzenesulfonyl chloride could initiate the polycondensation in pyridine but not in tetrahydrofuran (THF). It was found that glycine was subjected to a polycondensation reaction in the presence of benzenesulfonyl chloride in pyridine to form polypeptide having a low solution viscosity. More detailed investigations on the optimum conditions to form a high molecular weight polyamide are being carried out and results will be published in the near future.

\section{REFERENCES}

1. N. Ogata, K. Sanui, and K. Iijima, J. Polym. Sci., Polym. Chem. Ed., 11, 1096 (1973).

2. B. Iselin, W. Rittle, P. Sieber, and R. Schwyzer, Helv. Chim. Acta, 40, 373 (1957).

3. W. E. Bissinger and F. E. Kung, J. Am. Chem. Soc., 70, 2664 (1948).

4. C. A. Bunton and G. Schwerin, J. Org. Chem., 31, 843 (1965). 